Research Paper

\title{
Pregnancy Specific $\beta-1$ Glycoprotein 1 is Expressed in Pancreatic Ductal Adenocarcinoma and its Subcellular Localization Correlates with Overall Survival
}

\author{
Jasmin H. Shahinian ${ }^{1}$, Hannah Fuellgraf ${ }^{2}$, Stefan Tholen ${ }^{3}$, Justin Mastroianni ${ }^{5}$, Julia Daniela Knopf ${ }^{3,4}$, \\ Markus Kuehs, ${ }^{2}$, Bettina Mayer ${ }^{3}$, Manuel Schlimpert ${ }^{3}$, Birte Kulemann' ${ }^{6}$, Simon Kuesters ${ }^{6}$, Jens \\ Hoeppner ${ }^{6,9}$, Ulrich F. Wellner7, Martin Werner2,9,10, Ulrich T. Hopt ${ }^{6}$, Robert Zeiser ${ }^{5,8}$, Peter Bronsert ${ }^{2,9,10}$, \\ Oliver Schilling $3,8,10 \bowtie$ \\ 1. Division of Cardiac Surgery, University Hospital Basel, Basel, Switzerland; \\ 2. Institute of Surgical Pathology, University Medical Center Freiburg, Germany; \\ 3. Institute of Molecular Medicine and Cell Research, University of Freiburg, Germany; \\ 4. present address: Zentrum für Molekulare Biologie der Universität Heidelberg (ZMBH), DKFZ-ZMBH Allianz, Im Neuenheimer Feld 282, 69120 Heidelberg, \\ Germany; \\ 5. Department of Hematology and Oncology, University Medical Center Freiburg, Germany; \\ 6. Clinic for General and Visceral Surgery, University Medical Center Freiburg, Germany; \\ 7. Clinic for Surgery, University Clinic Schleswig-Holstein Campus Lübeck, Germany; \\ 8. BIOSS Centre for Biological Signaling Studies, University of Freiburg, D-79104 Freiburg, Germany; \\ 9. Comprehensive Cancer Center Freiburg, Germany; \\ 10. German Cancer Consortium (DKTK) and German Cancer Research Center (DKFZ), Heidelberg, German.
}

$\bowtie$ Corresponding author: Stefan-Meier-Str. 17, D-79104 Freiburg, Germany.+49 7612039615 oliver.schilling@mol-med.uni-freiburg.de.

(C) Ivyspring International Publisher. Reproduction is permitted for personal, noncommercial use, provided that the article is in whole, unmodified, and properly cited. See http://ivyspring.com/terms for terms and conditions.

Received: 2016.04.16; Accepted: 2016.09.18; Published: 2016.10.17

\begin{abstract}
Proteins of the pregnancy specific $\beta-1$ glycoprotein (PSG) family are renowned for their elevated expression during pregnancy. Only few reports have investigated their expression in adenocarcinomas. We studied the expression of PSG1 in pancreatic adenocarcinoma (PDAC). In a cohort of 104 patient samples, immunohistochemical analysis determined PSG1 expression in every specimen. PSG1 was found at apical and cytoplasmic localization or solely at cytoplasmic localization, with the latter case being correlated to shortened median survival ( 25 vs 11 months, logrank p-value $<0.001$ ). At the same time, enzyme linked immunosorbent assay (ELISA) did not detect elevated PSG1 levels in the plasma of PDAC patients as opposed to the plasma of healthy, non-pregnant control individuals. We also probed the impact of PSG1 expression in a murine tumor model system, using subcutaneous injection of Colo-26 cells into immunocompetent $\mathrm{BALB} / \mathrm{c}$ mice. Here, tumor growth was not affected by the expression of human PSG1. Our study reaffirms interest into the tumor-contextual biology of PSG proteins.
\end{abstract}

Key words: Pregnancy Specific $\beta-1$ Glycoprotein, Schwangerschaftsprotein, Immunohistochemistry, Subcellular localization.

\section{Introduction}

Members of the pregnancy specific glycoprotein family (PSGs) were first described in the early 1970s $[1,2]$. In vitro, PSGs have been identified in cultured trophoblasts [3]. The human genome encodes for 11 PSGs [4], many of which undergo differential mRNA splicing, thus yielding a larger number of protein variants [5]. PSGs are mostly renowned for their abundant placental expression during pregnancy [2], which results in elevated PSG plasma levels. Below-average PSG plasma levels in pregnant women have been associated with "small for gestation age" (SGA) fetuses [6] and first-trimester maternal plasma levels of PSG1 have been shown to be influenced by smoking [7]. 
In contrast to members of the related, membrane-attached carcinoembryonic antigen (CEA) family, PSGs are secreted glycoproteins. Physiologically, PSGs are predominantly produced in syncytiotrophoblasts [8]. The PSG plasma level during pregnancy increases continuously until a plateau is reached around the 36th week of gestation $[9,10]$.

PSGs have been occasionally reported to be expressed in adenocarcinomas and have been discussed as putative onco-fetal biomarker for breast $[11,12]$ and lung cancer [13] [14], and for urothelial neoplasm [15]. Very recently, PSG expression has also been reported for squamous cell carcinomas and colorectal cancers [16].

PSG consist mostly of one immunoglobulin variable-like domain and two - three immunoglobulin constant-like domains [1, 2, 17]. In many cases, PSGs localize to the cell surface by interacting with further cell surface proteins. This includes binding of selected PSGs to members of the tetraspanin family, surface-expressed glycosaminoglycans, and various integrins (reviewed in [17])

PSGs are proposed to function as regulators of the innate and adaptive immune response [18]. Current data suggest that PSGs participate in modulating the adaptive T-cell response. PSGs are further suggested to interact with antigen presenting cells as well as influencing the spectrum of cytokines and chemokines secreted by monocytes and macrophages. In addition, a pro-angiogenic function of PSGs has been suggested as well as a role in regulating the activity and availability of transforming growth factor (TGF)- $\beta 1$ and TGF- $\beta 2$ [17].

Pancreatic cancer is the fourth most common internal cancer in men and women in Europe, with almost 80,000 deaths annually [19]. Taken worldwide, there is an annual incidence of over 200,000 pancreatic cancer cases with an annual mortality that is almost equal to the incidence rate [20]. Pancreatic cancer is an aggressive malignancy with one of the worst outcomes of all cancers and has a very poor prognosis: with a 1-year survival of less than $25 \%$, and once the tumors have metastasized, a five-year survival of less than 5\% [21]. Tumors evolve numerous mechanisms to escape from immune recognition [22]. Regulatory immune cells such as monocytes and neutrophils have been reported [23] to play a crucial role for PDAC immune escape and tumor aggressiveness. In the present study, we present one of the first analyses of PSG1 expression in a pancreatic cancer cohort.

\section{Materials and Methods}

\section{Ethics statement}

The study was approved by the Ethics Committee of the Medical University Freiburg, (13/11, "invasion and metastasis of periampullary cancers"). Before study inclusion, all patient data were pseudonymized.

\section{Cell culture and transduction}

Colo-26 cells were provided by Prof. Zeiser, Freiburg, Germany. BxPC3 cells were purchased from ATCC. Cells were cultured in Dulbecco's modified eagle medium (DMEM; PAN Biotech) supplemented with $10 \%$ fetal calf serum (FCS; PAN), $1 \%$ non-essential amino acids, $1 \%$ MEM vitamins, $1 \%$ penicillin/streptomycin, and $10 \mathrm{mM}$ HEPES ( $\mathrm{pH} 7.5$ ) at $37{ }^{\circ} \mathrm{C}$ in humidified air containing $5 \% \mathrm{CO}_{2}$. Serum-free cell-conditioned medium (CCM) of human $\mathrm{BxPC} 3$ pancreatic ductal adenocarcinoma cells for western-blot analysis (see below) was generated as described previously for the ovarian cancer cell line OV-MZ-6 [24]. Murine Colo-26 colon cancer cells were transduced for stable luciferase expression as described previously for 291PC Burkitt lymphoma cells [25]. These cells were further transduced with the vector pReceiver-Lv105, constitutively expressing untagged human PSG1 under control of the cytomegalovirus (CMV) promoter (Genecopoeia, EX-Z8117-Lv105). pReceiver-Lv105 lacking the PSG1 gene served as an empty vector control (Genecopoeia, EX-NEG-Lv105). Puromycin selection $(20 \mu \mathrm{g} / \mathrm{ml}$ for $14 \mathrm{~d})$ was used for selection of stable transfectants. Serum-free cell-conditioned medium (CCM) of human $\mathrm{BxPC} 3$ pancreatic ductal adenocarcinoma cells and Colo-26 cells for western-blot analysis (see below) was generated as described previously for the ovarian cancer cell line OV-MZ-6 [24].

\section{Western Blot}

Cryopreserved tissues (normal pancreas, PDAC, placenta) were lysed in $10 \mathrm{mM}$ Na-ethylenediaminetetraacetate, $\mathrm{pH} 8.0 ; 1 \mathrm{mM}$ phenlymethansulfonylflouride; $1 \mu \mathrm{M}$ trans-epoxysuccinylL-leucylamido(4-guanidino)butane using a Precellys homogenizer (ceramic beads 1.4 / $2.8 \mathrm{~mm}$; $6500 \mathrm{rpm}$ for up to four intervals of $2 \mathrm{~min}$; Peqlab). Lysates were centrifuged for $10 \mathrm{~min}$ at $16600 \mathrm{x} \mathrm{g}, 4{ }^{\circ} \mathrm{C}$. The supernatant was used for further analysis. Protein concentration was determined using the Bradford method (Bio-Rad). Samples (10 $\mu \mathrm{g}$ of protein) were separated by SDS-PAGE and transferred on polyvinylidene fluoride (PVDF) membrane using a semidry blotting system (BioRad), followed by blocking with $4 \%$ non-fat milk in PBS-Tween and 
incubation with the primary mouse monoclonal antibody targeting human PSG1 (18 h at $4^{\circ} \mathrm{C}, 1: 500$, R\&D Systems, MAB6799). After washing, the membranes were incubated for $18 \mathrm{~h}$ at $4^{\circ} \mathrm{C}$ with the corresponding secondary anti-mouse antibody (Dianova, Hamburg, Germany \#115-035-003). The membranes were washed and developed with the West Pico Chemiluminescent substrate (Pierce, Rockford, USA). Peroxidase activity was detected with the Fusion SL device (Peqlab, Erlangen, Germany). Deglycosylation was performed according to manufacturer's instructions ("Protein Deglycosylation Mix", New England Biolabs, Ipswich, MA, USA).

\section{Patients and tumor tissue}

All patients were treated between 2007 and 2011 at the Clinic for General and Visceral Surgery, University Medical Center Freiburg, Germany for PDAC. Histopathological work-up was performed at the Institute of Surgical Pathology, University Medical Center Freiburg, Germany. All histological samples from the tumor were revalidated independently by two experienced pathologists (P.B., H.F.). Clinical data from the database of the Clinic for General and Visceral Surgery, University Medical Center Freiburg, Germany were reviewed by S.K. and J.H. for correctness. In total, 104 patients were initially included.

\section{Standard pathology work-up}

From a prospectively maintained database, patients with PDAC were selected. For all specimens a standardized gross examination and histopathological work-up [26] was performed. Frozen section analysis, examined by experienced pathologists, was performed for all specimens at the Institute of Surgical Pathology, University Medical Center Freiburg, Germany. Tumor-masses were measured in all three dimensions; staging parameters (tumor size, tumor grade (G1 - G4), absence or presence of lymphatic (L0 / L1), blood vessel (V0 / V1), perineural invasion (Pn0 / Pn1) and local lymph node (N0 / N1) infiltration) were documented and classified according to the latest UICC classification. Tumor histology was grouped according to the latest WHO Classification. The parenchymal and deep resection margins, the oral and aboral duodenal resection margins as well as the resection margin of the Ductus choledochus were exanimated macro- and microscopically for the absence (R0) or presence (R1) of tumor cells.

\section{PSG immunohistochemistry}

For immunohistochemical PSG analysis, from selected suitable formalin fixed and paraffin embedded tissue samples, slices of $2 \mu \mathrm{m}$ thickness were prepared using the Leica RM2255 microtome. Antigen retrieval was performed for 30 minutes with DAKO Retrieval Solution S1699 in a Braun steamer and cooled down onto ice for 20 minutes afterwards. Next, incubation with PSG primary antibody ((PSG) Clone \# 684701 Catalog Number: MAB6799) for 30 minutes diluted 1:1000 in Zytomed dilution buffer (ZUC025-500) was conducted. Visualization was performed using DAKO detection system K5005. Sections were counterstained with haemalm for one minute, dehydrated in an ascending alcohol concentration and covered with xylol and coverslipping film (Tissue-Tek ${ }^{\mathrm{R}}$ 4770).

PSG evaluation: Two experienced pathologists (HF, PB), blinded for clinico-pathological parameters, reviewed PSG expression according to the following protocol. Using 200-fold magnification, PSG expression was analyzed performing a semi-quantitative expression analyses by evaluating and multiplying PSG intensity (range 0 to 3 ) and the percentage of PSG-positive (range 0-100 \%, intervals of $5 \%$ ) tumor cells. In detail, if no signal was detectable the PSG expression was scored negative (Score 0). If a slack PSG signal was detectable score 1 (low intensity) was used. If PSG was expressed higher than score 1, but the haematoxylin counterstained nucleus demonstrated a higher staining intensity than PSG, score 2 (moderate intensity) was used. Score 3 (high intensity) was used if PSG intensity was higher than the haematoxylin counterstained nucleus. Furthermore, PSG localization "luminal and intracellular" and intracellular only was assessed. For all analyses, the percental predominant score was used for adjacent statistical analyses. Placental tissue was used as a positive control. Normal, non-malignant pancreatic tissue only demonstrates a very faint cytoplasmic expression of PSG in the exocrine cells.

Statistical analysis: IBM SPSS Statistics Version 21 (SPSS Inc. Chicago, IL) was used for statistical analyses. Survival data were analyzed using the Kaplan-Meier method and the Logrank test. Univariate analyses were performed via Spearman Chi squared test. For multivariate analyses, clinico-pathological predictors reaching a trend significance level $(p<0.15)$ were included into a Cox proportional hazards model using forward and backward analyses. All statistical tests were performed two-sided. Significance level was set to $\mathrm{p}<0.05$.

\section{Enzyme-linked Immunosorbent Assay (ELISA)}

ELISA for PSG1 concentration in EDTA-plasma samples was performed according to manufacturer's 
instructions (R\&D Systems, Human PSG1 Quantikine ELISA Kit), based on a monoclonal antibody specific for human PSG1. EDTA-plasma of patients was collected preoperatively.

\section{Murine Tumor Model}

BALB/c mice were purchased from the local stock of the animal facility at Freiburg University Medical Center. Mice were used between 6 and 12 weeks of age. On day 0, $2.0 \times 10^{5}$ luciferase expressing Colo-26 cells (see above) containing a human PSG1 overexpression vector $(\mathrm{n}=3)$ or empty vector control $(n=3)$ were injected subcutaneously into the flanks of mice. Tumor volume was then monitored via bioluminescence imaging and palpation, with a weekly and alternating daily schedule, respectively. Maintenance of animal strains and work performed in this study was carried out in accordance with institutional guidelines and the German law for animal protection (Tierschutzgesetz) as published on May 18th 2006 with last amendment on July 28th 2014. Ethics approval registration number is G-13/116 RP regional council Freiburg.

\section{Results and Discussion}

\section{Public PDAC Expression Datasets Suggest a Weak Association Between Elevated PSG 1 Expression and Shortened Survival}

There have been a handful of reports on the expression of PSG proteins (sometimes also referred to as "Schwangerschaftsprotein" (SP), which has been used as a synonym) in epithelial cancers [11-13, 27-32]; however, PSG expression in PDAC has not yet been investigated. Based on these findings we explored publicly accessible PDAC gene expression datasets for a putative association between PSG1 mRNA expression and cumulative survival. Both the study of Stratford et al. [33] and Zhang et al. [34], probed using the Survexpress [35] and Precog [36] platforms, highlighted a trend for decreased survival in correlation with increased PSG1 expression (Supplementary Figure 1). In both cases, the p-value (log-rank test) did not meet statistical significance $(\mathrm{p}=$ 0.27 and $p=0.11$, respectively). However, since a similar trend was observed in two independent studies, we conclude that there is a weak association between elevated PSG1 expression and shortened survival.

\section{Detection of PSG 1 by Immunoblotting in PDAC Specimens}

In a next step, we used immunoblotting to probe for PSG1 expression in PDAC specimens. A commercially available, monoclonal antibody against human PSG1 detects a band at approximately $75 \mathrm{kDa}$, which is present in PDAC specimens and absent in normal pancreatic tissue (Figure 1). The same band was detected in placenta tissue and conditioned medium of human BxPC3 PDAC cells (Figure 1). The $\sim 75 \mathrm{kDa}$ band is absent in conditioned medium of wild-type murine Colo-26 cells but overexpression of human PSG1 leads to its appearance. Since PSG1 is reported to be glycosylated and the detected molecular weight of $\sim 75 \mathrm{kDa}$ exceeds the $\sim 380$ residue length of mature PSG1, we also probed for deglycosylated PSG1. Upon deglycosylation, the molecular weight shifts to approximately $37 \mathrm{kDa}$, in line with the $\sim 380$ residue length of mature PSG1.

PSG proteins belong to the carcinoembryonic antigen (CEA) family [17]. Carcinoembryonic antigen-related cell adhesion molecule (CEACAM)-5 is the prototypic member of the CEA family. Elevated expression of CEACAM5 in solid tumors has been reported as early as 1965 [37] and CEACAM5 plasma levels are nowadays used for the diagnostic monitoring of several cancers, including PDAC [38, 39]. CEACAM 5 is typically detected with a molecular weight $>150 \mathrm{kDa}$ by immunoblotting [39]. In our PDAC samples, we detect PSG1 at approximately 75 $\mathrm{kDa}$.

However, some members of CEACAM family have been detected in the molecular weight range around $70 \mathrm{KDa}$, e.g. CEACAM6 [40]. With regard to putative antigen cross-reactivity with CEACAMs, it is worth noting that we detect a single strong band in western-blot analysis, and not multiple strong bands, e.g. stemming from cross-reactivity with multiple CEACAM proteins. Regarding CEACAM6, its mature form consists of less than 290 residues with extensive glycosylation yielding a $64 \mathrm{kDa}$ products. In our study, deglycosylation yields a $\sim 37 \mathrm{kDa}$ product, which is too large for a $<290$ residue protein. The differences in molecular weight further support detection of PSG1. Nevertheless, as for any antibody-based method, we acknowledge a vague and generally applicable possibility of antigen cross-reactivity.

Furthermore, two global transcriptome studies comparing PDAC with non-malignant pancreatic tissue corroborate our finding of increased PSG1 expression PDAC. The studies of Logsdon et al [41] and Iacobuzio-Donahue et al [42] both report significantly elevated PSG1 transcript levels in PDAC ( $\mathrm{p}<0.01$, two-tailed student t-test).

In summary, our immunoblotting data supports tumor-associated expression of PSG1 in PDAC. 

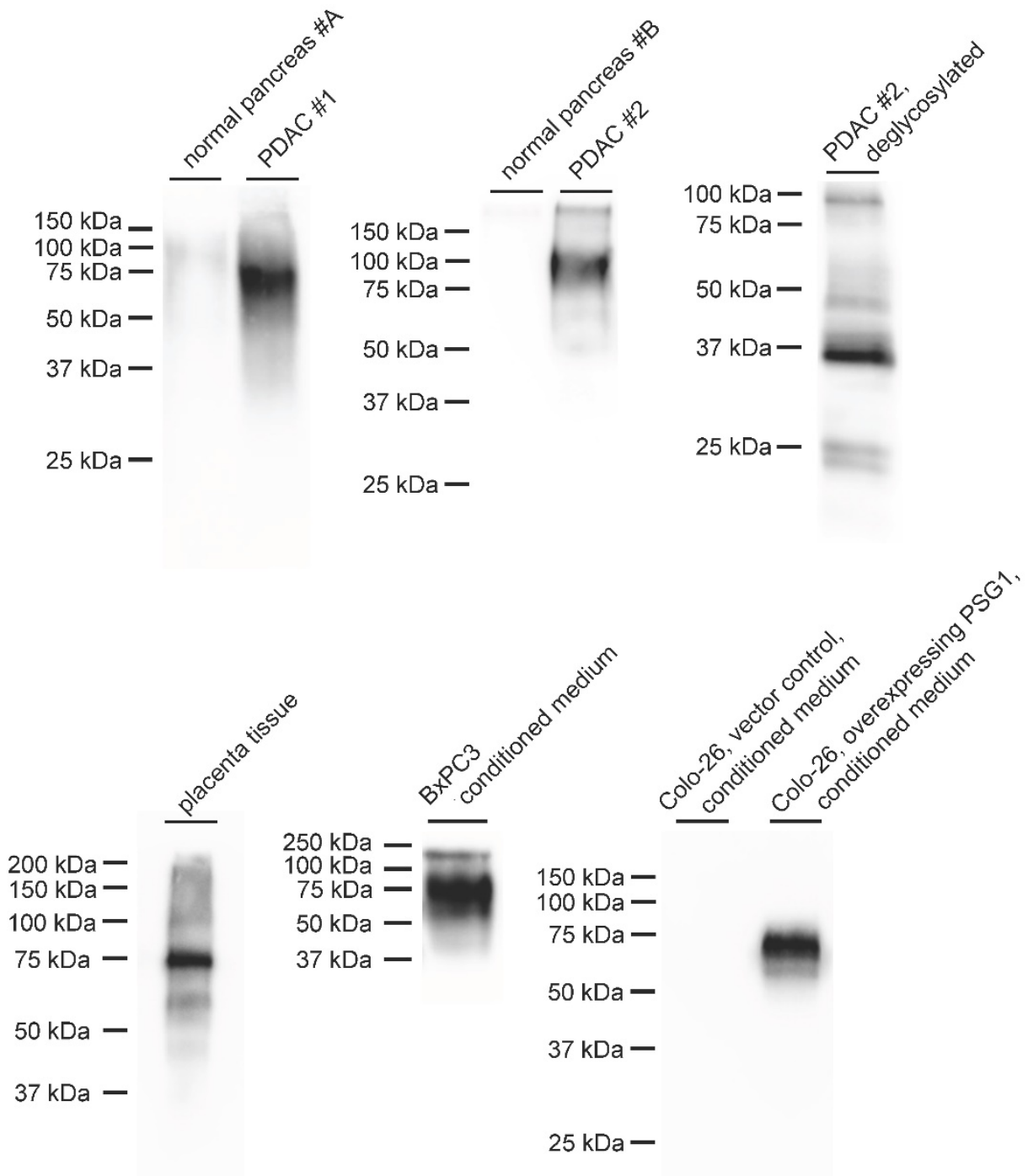

$25 \mathrm{kDa}-$

Figure 1: Immunoblot detection of PSG1 using a commercially available monoclonal antibody. In two cases, a strong signal between 75 and $100 \mathrm{kDa}$ is observed in samples from pancreatic adenocarcinoma (PDAC), which is missing in non-malignant pancreatic tissue. Deglycosylation yields a signal of approximately $37 \mathrm{kDa}$. Immunoblot detection of PSG 1 in placenta tissue equally yielded a strong signal at $75 \mathrm{kDa}$, which was also detected in conditioned medium of cultured BxPC3 PDAC cells and in murine Colo-26 cells that were stably transfected to produce human PSG1.

\section{Immunohistochemical Analysis of PSG1 Expression in PDAC}

Baseline parameters: Next we analyzed the impact of PSG expression levels and cellular localization on overall survival in a cohort of 104 adjuvant-treated PDAC patients. Median age was 66 years, 54 patients were female, 50 patients' male. 90 patients received a pylorus preserving pancreaticoduodenotomie (PPPD), 13 a Whipple operation and one patient a total pancreatectomy. Median tumor size was $28 \mathrm{~mm}$ (range $3-60 \mathrm{~mm}$ ). According to UICC-Classification four patients were classified as T1 $(4 / 104 ; 3.8 \%)$, six T2 (6 / 104; $5.8 \%), 88$ T3 (88 / 104; $84.6 \%)$ and six T4 (6 / 104; $5.8 \%$ ). Lymph node status was assessed for all patients. Thereby 27 patients $(26 \%)$ were nodal negative (N0), 77 (74\%) were nodal positive. Lymphangioinvasion (L1) was observed in 51 (49\%), haemangioinvasion (L1) in 20 (19 \%), perineuralinvasion (Pn1) in 79 (76\%) and distant metastases (M1) in 3 (3\%) patients. Regarding tumor differentiation, three tumors were well $(3$ / 104; 2.9 $\%), 58$ moderately (58 / 104; $55.8 \%$ ), 42 poorly (42 / $104 ; 40.4 \%)$ and one un-differentiated (1 / 104; $1 \%)$. One resection margin $(1 / 104 ; 1 \%)$ was macroscopically, 36 resection margins (36 / 104; $34.6 \%$ ) were microscopically positive and 67 resection margins $(67 / 104 ; 64.4 \%)$ were microscopically negative for tumor cells.

\section{PSG I immunohistochemistry}

PSG1 expression was analyzed in all 104 PDAC samples. To this end, PSG1 expression was probed by immunohistochemistry as detailed in Materials and Methods. As a positive control, we initially probed placenta tissue and detected prototypical luminal 
PSG1 staining, which was predominantly restricted to luminally located syncytiotrophoblasts (Figure 2a). In line with the immunoblotting analysis, normal, non-malignant pancreatic tissue only demonstrates a very faint cytoplasmic expression of PSG in the exocrine cells. (Figure 2b). However, in all PDAC specimens some PSG1 staining was detected with no case of completely negative staining. 22 tumors demonstrated weak (22 / 104; $21.15 \%), 17$ (17/ 104; $16.35 \%)$ a moderate and $65(65 / 104 ; 62.5 \%)$ a strong staining intensity. PSG1 was expressed as a

a)

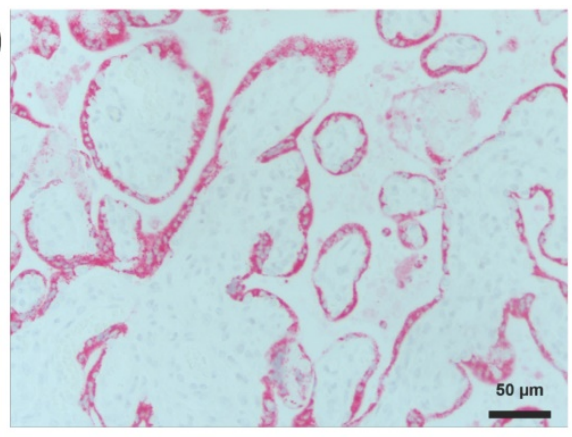

c)

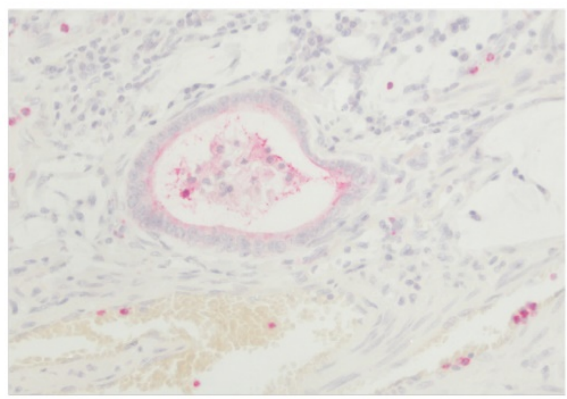

d)

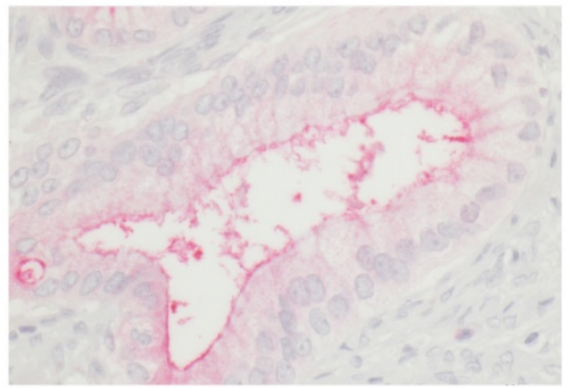

e)

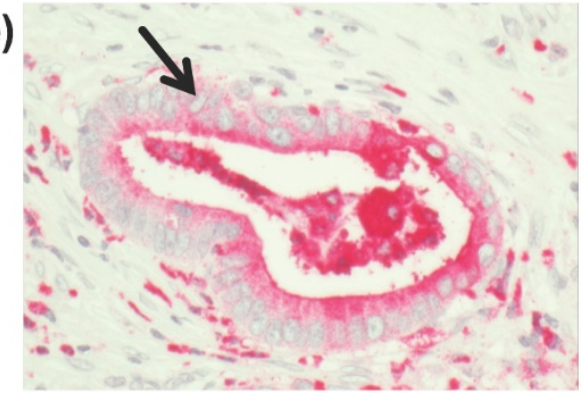

percentage range from 1 to $100 \%$ (mean $=77.56 \%)$. Importantly, two types of staining were distinguished, based on the subcellular localization of intense PSG1 staining:

a) predominantly apical and partially cytoplasmic (prototypical cases shown in Figure 2c e), referred to as "apical-cytoplasmic"

b) predominantly cytoplasmic (prototypical cases shown in Figure $\mathbf{2 f}-\mathbf{h}$ ), referred to as "cytoplasmic-only"

b)

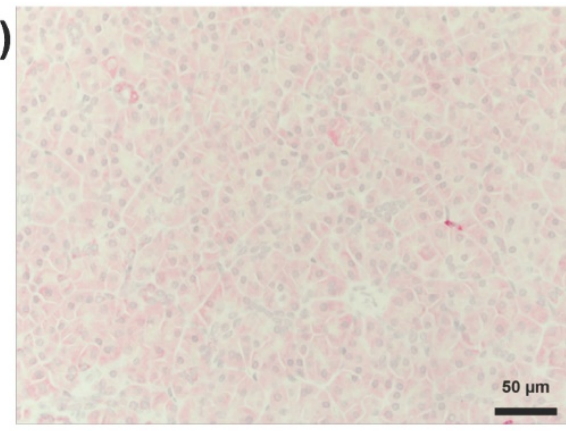

f)

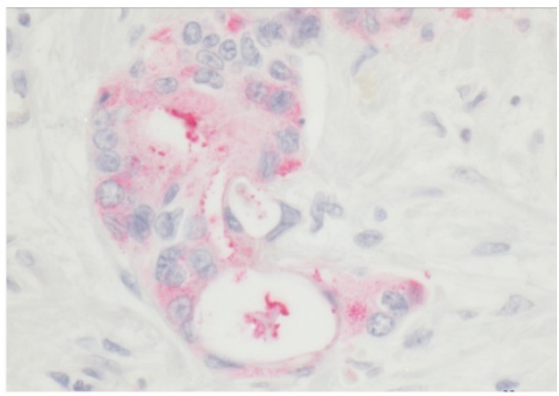

g)

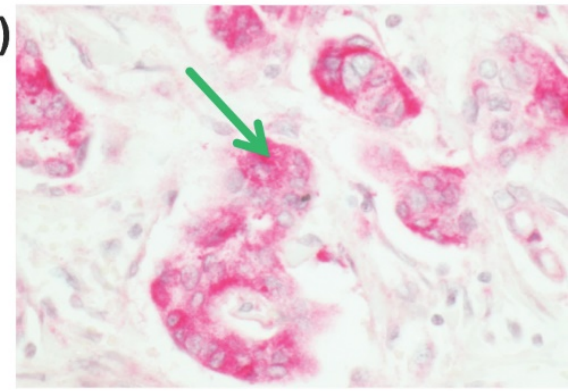

h)

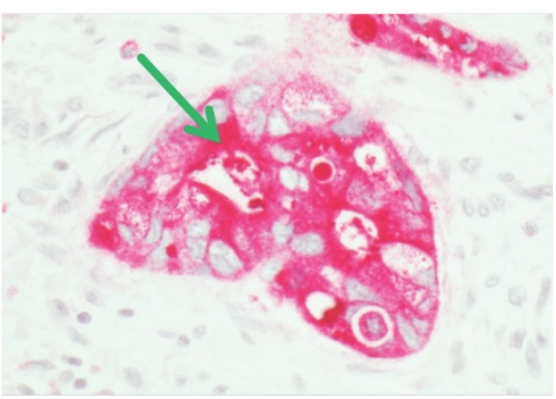

Figure 2: Immunohistochemical analysis of PSG1. Representative pictures are shown. (a) In placenta tissue, a prototypical luminal PSG1 localization is detected. (b) Normal, non-malignant pancreatic tissue only demonstrates a faint cytoplasmic expression of PSG in the exocrine cells. In PDAC tissue, substantial PSG1 levels are detected, with two types of distinct subcellular localizations: (c - e) predominantly apical (black arrow) and ( $\mathrm{f}-\mathrm{h})$ predominantly cytoplasmic (green arrows); each at low (c, f), moderate (d, g), and high (e, h) PSGl expression. (c - h) were recorded at 400 fold magnification. 
$31 \%(32 / 104)$ of all tumors were "cytoplasmic-only" PSG1 positive, whereas 69 \% (72 / 104) of all tumors were "apical-cytoplasmic "PSG1 positive. Several earlier publications have already demonstrated a seemingly intracellular / cytoplasmic staining for PSG proteins in adenocarcinoma specimens [11, 28, 29] and normal syncytiotrophoblast cells $[8,43]$. Differential subcellular localization in a disease setting has been previously reported for other proteins such as the cell surface protease prostasin in dermatitis [44].

\section{Statistical analyses}

Univariate analyses of our cohort highlighted metastatic lymph node ratio and surgical regime (pylorus preserving pancreatoduodenectomy vs. Whipple procedure vs. pancreatectomy) as significant, classical, clinico-pathological parameters for survival $(\mathrm{p}=0.022)$. This is in line with a previous report [45]. Statistical trends ( $\geq 0.05$ and $<0.15)$ were observed for perineural invasion $(p=0.116)$ and tumor grading $(p=0.107)$. Classical UICC relevant parameters (TNM classification, lymphangiosis, haemangiosis) as well as patients gender or age and R-classification demonstrated no significant correlation in univariate analyses. Considering PSG expression in the tumor cells, neither PSG intensity nor percentage correlated with overall survival $(\mathrm{p}=$ 0.0.744 and $\mathrm{p}=0.319)$. However, "cytoplasmic-only" versus "apical-cytoplasmic" PSG1 expression ( $\mathrm{p}<$ $0.001)$ was highly significant in univariate analyses (Figure 3) with "cytoplasmic-only" staining being linked to shorter cumulative survival. Details of the univariate analysis are shown in Table 1.

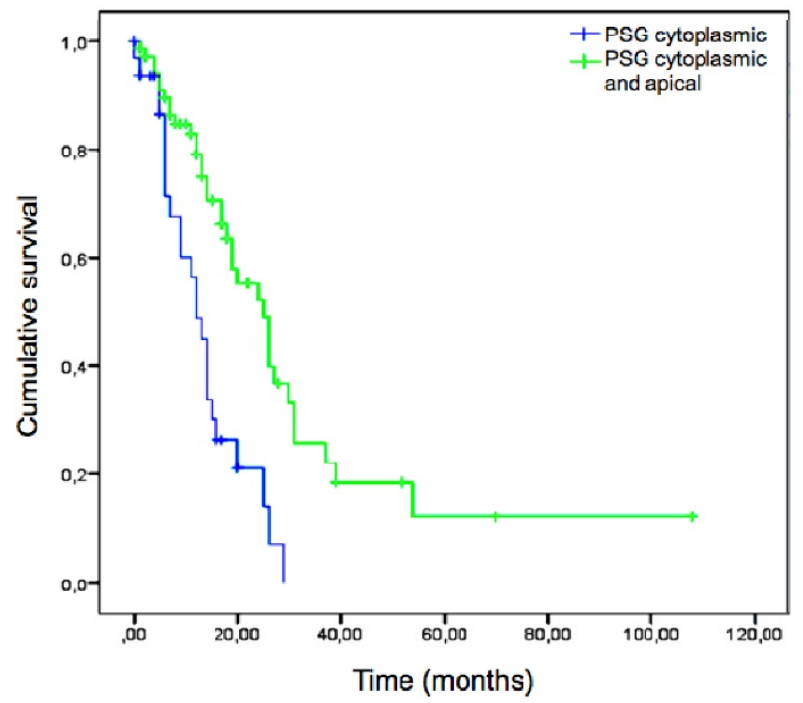

Figure 3: Kaplan-Meier plot depicting overall survival of PDAC patients $(n=104)$ according to PSG1 localization ("cytoplasmic and apical" in green or "cytoplasmic only" in blue). "Cytoplasmic only" localization correlated to shorter overall survival (log-rank p-value <0.001).
Table 1. Univariate and multivariate survival analysis.

\begin{tabular}{|c|c|c|c|c|c|c|c|}
\hline Parameter & Condition & $\mathbf{N}$ & $\begin{array}{l}\text { Events } \\
\text { (deaths) }\end{array}$ & $\begin{array}{l}\text { Median } \\
\text { survival } \\
\text { (months) }\end{array}$ & $\begin{array}{l}\text { Logrank } \\
\text { p }\end{array}$ & $\begin{array}{l}\text { Hazard } \\
\text { Ratio } \\
(95 \% \text { CI })\end{array}$ & $\begin{array}{l}\text { Cox } \\
\mathrm{p}\end{array}$ \\
\hline All patients & & 104 & 59 & 19 & & & \\
\hline \multirow[t]{2}{*}{ Gender } & male & 50 & 29 & 18 & 0.869 & NI & \\
\hline & female & 54 & 30 & 19 & & & \\
\hline \multirow[t]{2}{*}{ Age ${ }^{1}$} & $<67$ & 55 & 31 & 20 & 0.532 & NI & \\
\hline & $>=67$ & 49 & 28 & 17 & & & \\
\hline \multirow{2}{*}{$\begin{array}{l}\text { Neoadjuvant } \\
\text { therapy }\end{array}$} & no & 95 & 52 & 19 & 0.250 & NI & \\
\hline & yes & 9 & 7 & 17 & & & \\
\hline \multirow[t]{3}{*}{ Resection } & PPPD & 90 & 52 & 19 & $<0.001$ & & E. \\
\hline & Whipple & 13 & 6 & 24 & & & \\
\hline & total PE & 1 & 1 & 2 & & & \\
\hline \multirow[t]{2}{*}{ Tumor size (mm) } & $<25$ & 36 & 23 & 19 & 0.534 & NI & \\
\hline & $>=25$ & 64 & 33 & 20 & & & \\
\hline \multirow[t]{2}{*}{ pT Stage } & $\mathrm{pT} 1 / 2$ & 10 & 7 & 20 & 0.740 & NI & \\
\hline & $\mathrm{pT} 3 / 4$ & 94 & 52 & 19 & & & \\
\hline \multirow[t]{2}{*}{ pN Stage } & pN0 & 26 & 13 & 19 & 0.881 & NI & \\
\hline & $\mathrm{pN} 1$ & 78 & 46 & 18 & & & \\
\hline \multirow[t]{2}{*}{ Lymph node ratio } & $<0.106$ & 52 & 27 & 25 & 0.022 & $0.271-$ & 0.010 \\
\hline & $>=0.106$ & 52 & 32 & 17 & & 6.653 & \\
\hline \multirow[t]{2}{*}{ Lymphangiosis } & L0 & 53 & 35 & 19 & 0.930 & NI & \\
\hline & L1 & 51 & 24 & 18 & & & \\
\hline \multirow[t]{2}{*}{ Hemangiosis } & V0 & 84 & 45 & 20 & 0.202 & NI & \\
\hline & V1 & 20 & 14 & 12 & & & \\
\hline \multirow{2}{*}{$\begin{array}{l}\text { Perineural } \\
\text { invasion }\end{array}$} & Pn0 & 25 & 19 & 14 & 0.116 & & E \\
\hline & Pn1 & 79 & 40 & 20 & & & \\
\hline \multirow[t]{2}{*}{ Grade } & $\mathrm{G} 1 / 2$ & 61 & 35 & 20 & 0.107 & & E \\
\hline & $\mathrm{G} 3 / 4$ & 43 & 24 & 14 & & & \\
\hline \multirow[t]{2}{*}{ Resection Margin } & R0 & 67 & 35 & 18 & 0.175 & NI & \\
\hline & $\mathrm{R}+$ & 37 & 24 & 19 & & & \\
\hline \multirow[t]{2}{*}{ Distant metastasis } & M0 & 101 & 53 & 19 & 0.158 & NI & \\
\hline & M1 & 3 & 3 & 6 & & & \\
\hline \multirow[t]{3}{*}{ PSG intensity } & Weak & 22 & 14 & 25 & 0.744 & NI & \\
\hline & Moderate & 17 & 8 & 19 & & & \\
\hline & Strong & 65 & 37 & 16 & & & \\
\hline \multirow[t]{2}{*}{ PSG percent } & $<80 \%$ & 66 & 34 & 32 & 0.319 & NI & \\
\hline & $>=80 \%$ & 36 & 25 & 13 & & & \\
\hline \multirow[t]{2}{*}{ PSG localisation } & $\begin{array}{l}\text { Solely } \\
\text { cytoplasmic }\end{array}$ & 32 & 25 & 11 & $<0.001$ & $\begin{array}{l}0.094- \\
12.583\end{array}$ & 0.001 \\
\hline & $\begin{array}{l}\text { Apical and } \\
\text { cytoplasmic }\end{array}$ & 72 & 34 & 25 & & & \\
\hline
\end{tabular}

In a second step, we included all univariate correlations with $\mathrm{p}<0.15$ for a Cox proportional hazards model calculation performing forward selection and backward elimination. This multivariate analysis corroborated "intracellular-only" versus "apical-cytoplasmic" PSG expression and lymph node ratio $(p=0.01)$ as independent predictors of survival after resection.

\section{PSGI ELISA}

We further probed PSG1 levels in plasma of healthy, pregnant ( $\mathrm{n}=2$, for control purposes only) and non-pregnant individuals $(\mathrm{n}=3)$ as well as PDAC patients $(\mathrm{n}=8)$ using a commercially available PSG1 ELISA. In the non-pregnant individuals and PDAC 
patients we detected PSG1 concentrations in the range of $0.0-1.2 \mathrm{ng} / \mathrm{ml}$ (Figure 4); in line with an earlier report [11]. We did not detect elevated plasma levels of PSG1 in PDAC patients compared to healthy, non-pregnant controls. This finding is also in line with an earlier report [11].

PSG1 Expression Does Not Affect Tumor Growth in an Immunocompetent Murine Model System

Furthermore, we attempted to probe the effect of PSG1 expression on tumor growth using an immunocompetent model system. To this end, we used murine Colo-26 cancer cells, which were injected subcutaneously into the flanks of immunocompetent BALB/c mice. Stable expression of human PSG1 was achieved by transducing Colo-26 cancer cells with an expression vector for human PSG1. The same expression vector, lacking the PSG1 gene served as the "empty vector" control. Usage of a highly efficient viral transduction system together with antibiotic selection ensured the generation of stable transfectants. Expression or absence of human PSG1 is shown in Figure 1. $2.0 \times 10^{5}$ Colo-26 cells were injected subcutaneously into the flanks of BALB/c mice. Tumor growth was monitored by palpation (Figure 5a) or bioluminescence (Figure $\mathbf{5 b}$ ). In both cases, an impact of PSG1 expression on tumor growth was not detected. To our knowledge, this is one of the first in vivo studies focusing on the tumor-contextual function of a PSG protein.

a)

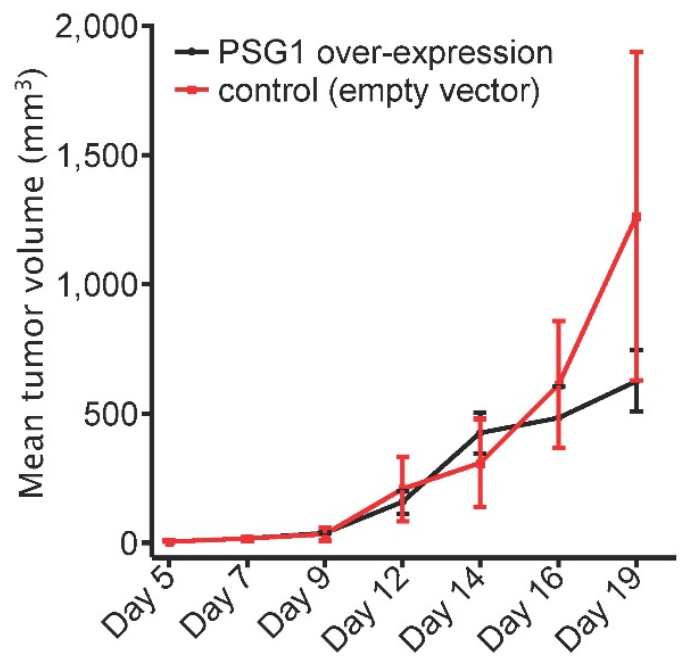

\section{Conclusion}

We present the first immunohistochemical analysis of PSG1 expression in PDAC. Our findings show "cytoplasmic-only" presence of PSG1 (as opposed to "apical-cytoplasmic") is associated with shorter cumulative survival after resection. At the same time, we did not detect elevated PSG1 levels in the plasma of PDAC patients nor did PSG1 expression affect tumor growth in an immunocompetent murine model system. In combination, these results underline that the role of the PSG system in PDAC biology warrants further investigation.

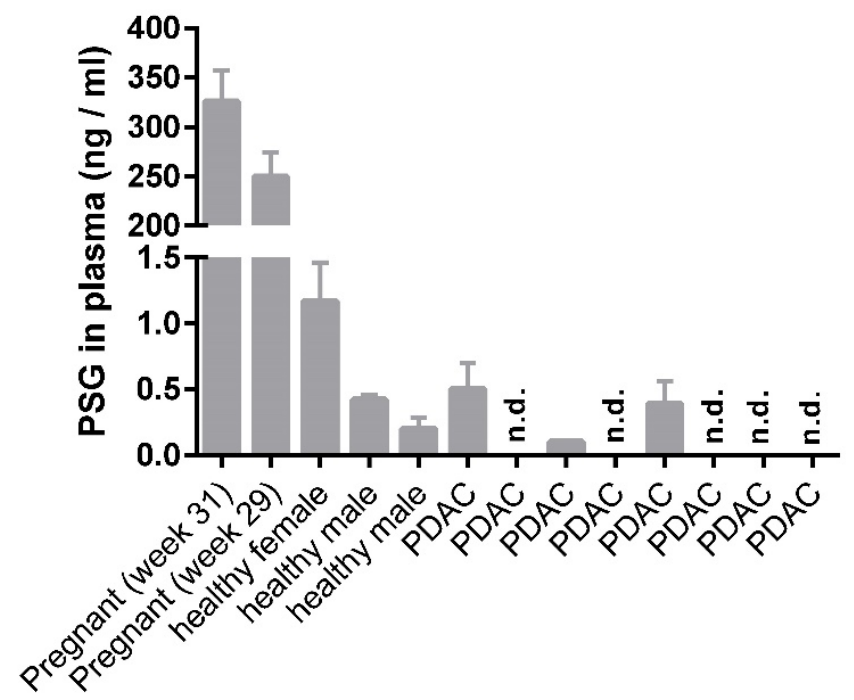

Figure 4: ELISA detection of PSG1 in plasma samples of healthy, pregnant and non-pregnant individuals and eight PDAC patients. n.d., not detected.

b)

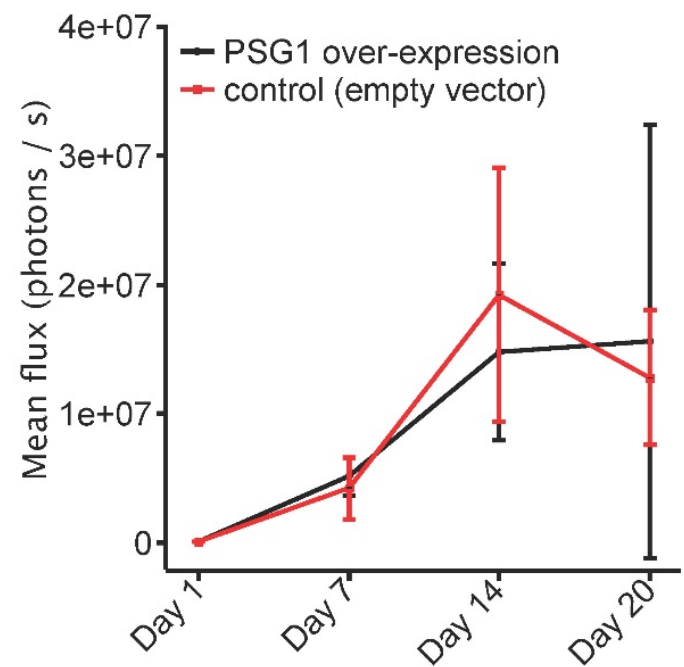

Figure 5: Tumor growth of Colo-26 cells expressing human PSG1 or corresponding Colo-26 control cells, subcutaneously injected into the flanks of immunocompetent BALB/c mice $(n=3$, each). Tumor growth was monitored by $(\mathbf{a})$ palpation or (b) bioluminescence. 


\section{Supplementary Material}

Supplementary figure 1.

http://www.jcancer.org/v07p2018s1.pdf

\section{Acknowledgement}

We thank the SFB50 Z1 project members Prof. Dr. Silke Lassmann and Nicola Bittermann for initial establishment of stainings. This study was funded by the Deutsche Forschungsgemeinschaft (SCHI 871/2 and SCHI $871 / 5$, SCHI $871 / 6$, GR 1748/6, INST 39/900-1 and SFB850-Project B8 to O. S., WE 5085/1-1 to U. W.), the European Research Council (ERC-2011StG 282111-ProteaSys to O.S.), and the Excellence Initiative of the German Federal and State Governments (EXC 294, BIOSS to O.S.). The article processing charge was funded by the German Research Foundation (DFG) and the University of Freiburg in the funding programme Open Access Publishing.

\section{Ethical Approval}

All procedures performed in this reciprocal study involving human participants were in accordance with the ethical standards of the institutional and/or national research committee and with the 1964 Helsinki declaration and its later amendments or comparable ethical standards. Also, all applicable international, national, and/or institutional guidelines for the care and use of animals were followed. Details are stated in the "Materials and Methods" section.

\section{Competing Interests}

The authors declare no competing interests.

\section{References}

1. Tatarinov Iu S, Masiukevich VN. [Immunochemical identification of a new beta-1-globulin in the serum of pregnant women]. Biull Eksp Biol Med. 1970; 69: $66-8$

2. Bohn H. [Isolation and characterization of pregnancy-specific beta1-glycoprotein]. Blut. 1972; 24: 292-302.

3. Horne $\mathrm{CH}$, Towler $\mathrm{CM}$, Pugh-Humphreys RG, Thomson AW, Bohn H. Pregnancy specific beta1-glycoprotein--a product of the syncytiotrophoblast. Experientia. 1976; 32: 1197

4. Teglund S, Olsen A, Khan WN, Frangsmyr L, Hammarstrom S. The pregnancy-specific glycoprotein (PSG) gene cluster on human chromosome 19: fine structure of the 11 PSG genes and identification of 6 new genes forming a third subgroup within the carcinoembryonic antigen (CEA) family. Genomics. 1994; 23: 669-84.

5. Teglund S, Zhou GQ, Hammarstrom S. Characterization of cDNA encoding novel pregnancy-specific glycoprotein variants. Biochem Biophys Res Commun 1995: 211: 656-64.

6. Pihl K, Larsen T, Laursen I, Krebs L, Christiansen M. First trimester maternal serum pregnancy-specific beta-1-glycoprotein (SP1) as a marker of adverse pregnancy outcome. Prenat Diagn. 2009; 29: 1256-61.

7. Pihl K, Christiansen M. Pregnancy-specific beta-1-glycoprotein in first-trimester maternal serum is influenced by smoking. Clin Chem. 2010; 56: 485-7.

8. Zhou GQ Baranov V, Zimmermann W, Grunert F, Erhard B, Mincheva-Nilsson L, et al. Highly specific monoclonal antibody demonstrates that pregnancy-specific glycoprotein (PSG) is limited to syncytiotrophoblast in human early and term placenta. Placenta. 1997; 18: 491-501.
9. Towler CM, Horne $\mathrm{CH}$, Jandial V, Campbell DM, MacGillivray I. Plasma levels of pregnancy-specific beta1-glycoprotein in normal pregnancy. Br J Obstet Gynaecol. 1976; 83: 775-9.

10. Wurz H, Geiger W, Kunzig HJ, Jabs-Lehmann A, Bohn H, Luben G. Radioimmunoassay of SP1 (pregnancy-specific beta1-glycoprotein) in maternal blood and in amniotic fluid normal and pathologic pregnancies. J Perinat Med. 1981; 9: 67-78.

11. Sorensen S, Andersen J, Norgaard T. Pregnancy-specific beta 1-glycoprotein (SP1) in serum and tissue from patients with benign and malignant breast tumours. Br J Cancer. 1984; 49: 663-7.

12. Cohen C, Sharkey FE, Shulman G, Uthman EO, Budgeon LR. Tumor-associated antigens in breast carcinomas. Prognostic significance. Cancer. 1987; 60: 1294-8.

13. Harach HR, Skinner M, Gibbs AR. Biological markers in human lung carcinoma: an immunopathological study of six antigens. Thorax. 1983; 38: 937-41.

14. Boucher LD, Yoneda K. The expression of trophoblastic cell markers by lung carcinomas. Hum Pathol. 1995; 26: 1201-6.

15. Campo E, Algaba F, Palacin A, Germa R, Sole-Balcells FJ, Cardesa A. Placental proteins in high-grade urothelial neoplasms. An immunohistochemical study of human chorionic gonadotropin, human placental lactogen, and pregnancy-specific beta-1-glycoprotein. Cancer. 1989; 63: 2497-504.

16. Houston A, Williams JM, Rovis TL, Shanley DK, O'Riordan RT, Kiely PA, et al. Pregnancy-specific glycoprotein expression in normal gastrointestinal tract and in tumors detected with novel monoclonal antibodies. MAbs. 2016: 0.

17. Moore T, Dveksler GS. Pregnancy-specific glycoproteins: complex gene families regulating maternal-fetal interactions. Int J Dev Biol. 2014; 58: 273-80.

18. Martinez FF, Cervi L, Knubel CP, Panzetta-Dutari GM, Motran CC. The role of pregnancy-specific glycoprotein 1a (PSG1a) in regulating the innate and adaptive immune response. Am J Reprod Immunol. 2013; 69: 383-94.

19. Malvezzi M, Bertuccio P, Levi F, La Vecchia C, Negri E. European cancer mortality predictions for the year 2012. Ann Oncol. 2012; 23: 1044-52.

20. Hariharan D, Saied A, Kocher HM. Analysis of mortality rates for pancreatic cancer across the world. HPB (Oxford). 2008; 10: 58-62.

21. Ellison LF, Wilkins K. An update on cancer survival. Health Rep. 2010; 21: $55-60$

22. Gajewski TF, Meng Y, Blank C, Brown I, Kacha A, Kline J, et al. Immune resistance orchestrated by the tumor microenvironment. Immunol Rev. 2006; 213: 131-45.

23. Mule JJ. Dendritic cell-based vaccines for pancreatic cancer and melanoma. Ann N Y Acad Sci. 2009; 1174: 33-40.

24. Shahinian H, Loessner D, Biniossek ML, Kizhakkedathu JN, Clements JA, Magdolen V, et al. Secretome and degradome profiling shows that Kallikrein-related peptidases 4, 5, 6, and 7 induce TGFbeta- 1 signaling in ovarian cancer cells. Mol Oncol. 2014; 8: 68-82.

25. Durr C, Pfeifer D, Claus R, Schmitt-Graeff A, Gerlach UV, Graeser R, et al. CXCL12 mediates immunosuppression in the lymphoma microenvironment after allogeneic transplantation of hematopoietic cells. Cancer Res. 2010; 70: $10170-81$.

26. Wellner UF, Krauss T, Csanadi A, Lapshyn H, Bolm L, Timme S, et al. Mesopancreatic Stromal Clearance Defines Curative Resection of Pancreatic Head Cancer and Can Be Predicted Preoperatively by Radiologic Parameters: A Retrospective Study. Medicine (Baltimore). 2016; 95: e2529.

27. Grudzinskas JG, Coombes RC, Ratcliffe JG, Gordon YB, Powles TJ, Neville $\mathrm{AM}$, et al. Circulating levels of pregnancy specific beta 1 glycopretein in patients with testicular, bronchogenic and breast carcinomas. Cancer. 1980; 45: $102-3$

28. Skinner JM, Whitehead R. Pregnancy-specific beta glycoprotein (SP1) in tumours of the human gastrointestinal tract. Br J Cancer. 1981; 44: 476-8.

29. Kuhajda FP, Bohn H, Mendelsohn G. Pregnancy-specific beta-1 glycoprotein (SP-1) in breast carcinoma. Pathologic and clinical considerations. Cancer. 1984; 54: 1392-6.

30. Gibbs AR, Harach R, Wagner JC, Jasani B. Comparison of tumour markers in malignant mesothelioma and pulmonary adenocarcinoma. Thorax. 1985; 40: 91-5.

31. Wright C, Angus B, Napier J, Wetherall M, Udagawa Y, Sainsbury JR, et al. Prognostic factors in breast cancer: immunohistochemical staining for SP1 and NCRC 11 related to survival, tumour epidermal growth factor receptor and oestrogen receptor status. J Pathol. 1987; 153: 325-31.

32. Salahshor S, Goncalves J, Chetty R, Gallinger S, Woodgett JR. Differential gene expression profile reveals deregulation of pregnancy specific beta1 glycoprotein 9 early during colorectal carcinogenesis. BMC Cancer. 2005; 5: 66.

33. Stratford JK, Bentrem DJ, Anderson JM, Fan C, Volmar KA, Marron JS, et al. A six-gene signature predicts survival of patients with localized pancreatic ductal adenocarcinoma. PLoS Med. 2010; 7: e1000307.

34. Zhang G, Schetter A, He P, Funamizu N, Gaedcke I, Ghadimi BM, et al. DPEP1 inhibits tumor cell invasiveness, enhances chemosensitivity and predicts clinical outcome in pancreatic ductal adenocarcinoma. PLoS One. 2012; 7: e31507.

35. Aguirre-Gamboa R, Gomez-Rueda H, Martinez-Ledesma E, Martinez-Torteya A, Chacolla-Huaringa R, Rodriguez-Barrientos A, et al. SurvExpress: an online biomarker validation tool and database for cancer gene expression data using survival analysis. PLoS One. 2013; 8: e74250. 
36. Gentles AJ, Newman AM, Liu CL, Bratman SV, Feng W, Kim D, et al. The prognostic landscape of genes and infiltrating immune cells across human cancers. Nat Med. 2015; 21: 938-45.

37. Gold P, Freedman SO. Specific carcinoembryonic antigens of the human digestive system. J Exp Med. 1965; 122: 467-81.

38. Beauchemin N, Arabzadeh A. Carcinoembryonic antigen-related cell adhesion molecules (CEACAMs) in cancer progression and metastasis. Cancer Metastasis Rev. 2013; 32: 643-71.

39. Hammarstrom S. The carcinoembryonic antigen (CEA) family: structures, suggested functions and expression in normal and malignant tissues. Semin Cancer Biol. 1999; 9: 67-81.

40. Strickland LA, Ross J, Williams S, Ross S, Romero M, Spencer S, et al. Preclinical evaluation of carcinoembryonic cell adhesion molecule (CEACAM) 6 as potential therapy target for pancreatic adenocarcinoma. J Pathol. 2009; 218: $380-90$.

41. Logsdon CD, Simeone DM, Binkley C, Arumugam T, Greenson JK, Giordano TJ, et al. Molecular profiling of pancreatic adenocarcinoma and chronic pancreatitis identifies multiple genes differentially regulated in pancreatic cancer. Cancer Res. 2003; 63: 2649-57.

42. Iacobuzio-Donahue CA, Maitra A, Olsen M, Lowe AW, van Heek NT, Rosty C, et al. Exploration of global gene expression patterns in pancreatic adenocarcinoma using cDNA microarrays. Am J Pathol. 2003; 162: 1151-62.

43. Schlafke S, Lantz KC, King BF, Enders AC. Ultrastructural localization of pregnancy-specific beta 1-glycoprotein (SP1) and cathepsin B in villi of early placenta of the macaque. Placenta. 1992; 13: 417-28.

44. Frateschi S, Camerer E, Crisante G, Rieser S, Membrez M, Charles RP, et al. PAR2 absence completely rescues inflammation and ichthyosis caused by altered CAP1/Prss8 expression in mouse skin. Nat Commun. 2011; 2: 161.

45. Paniccia A, Hosokawa P, Henderson W, Schulick RD, Edil BH, McCarter MD, et al. Characteristics of 10-Year Survivors of Pancreatic Ductal Adenocarcinoma. JAMA Surg. 2015; 150: 701-10. 